\title{
Emotional factors in robot-based assistive services for elderly at home
}

\author{
M. Díaz, J. Saez-Pons, M. Heerink and C. Angulo
}

\begin{abstract}
Emotional factors related to aging at home assistive technology are known to affect technology acceptance, effective use, and quality of life improvement. This paper is a survey on the affective dimension of robot-based systems conceived for helping elderly at home. The specificity of elders' capabilities (e.g. sensory and cognitive), coping styles, aspirations, lifestyles, social rules and preferences are faced with available knowledge from the fields of social psychology, sociology and gerontology. In the case of social robots, convenient verbal and non-verbal communication and motion behavior (e.g. social distance, space formations) are to be designed according to generational and cultural rules. Moreover, robot behavior should be congruent with its role (i.e. helper, companion) and affordances.
\end{abstract}

\section{INTRODUCTION}

Ethnographic research in the field of eldercare shows that the design of assistive devices determines their acceptance, efficient use, and intended improvement of elders' quality of life (QoL). The term aid technologies will be employed in this context, meaning any device, implementation, tool, computer program or supportive service designed to increase the capacities of people who, because of whatever circumstance, are not able to reach a predetermined competence [1].

However, it would be a misconception to assume that elders will use a technological aid just because they need it [2]. Understanding expectancies with regard to care and assistance of those interested - elders and their caregivers is necessary for effective assistive services and products design.

Relevant issues along the design life-cycle that can be assessed from empirical data are (1) whether the product is really helping or is disturbing users, (2) whether the system

* Research supported by the Spanish Ministry of Economy and Competitiveness through the project PATRICIA (TIN2012-38416-C03-01).

M. Díaz and C. Angulo are with CETpD Technical Research Center for Dependency Care and Autonomous Living UPC Technical University of Catalonia, Edifici Neàpolis, Rambla Exposició, 59-69, 08800 Vilanova i la Geltrú, Barcelona,Spain (e-mail: \{marta.diaz; cecilio.angulo\}@upc.edu)

J. Saez-Pons is with Adaptive Systems Research Group, School of Computer Science, University of Hertfordshire, Hatfield, United Kingdom (j.saez-pons@herts.ac.uk)

M. Heerink, is with La Salle, Ramon Llull University, Passeig Bonanova, 8 - 08022 Barcelona, SPAIN. Phone ++31-642917442, (e-mail: m.heerink@salle.url.edu) really makes elders feel competent or rather intimidated and stressed, and (3) whether elders feel proud of their achievements or rather ashamed to be depending on technology [3].

Some attributes of assistive technology design evoke certain emotions and perceptions, which influence the user's behavior. Hence, adoption degree or correct use can depend on the appearance of a device, affecting the general efficacy of the aid system, and in particular the user's health and QoL. Therefore, devices have to be not only useful but attractive. It is more probable for elderly to accept and adopt well designed devices because these cover simultaneously their functional, emotional and social needs [4].

Two questions arise from the consideration of emotional factors in robot-based systems: (1) which features of the assistive system influence perceptions and attitudes of older adults, and (2) how can these emotional factors be effectively addressed through design. In this context, social psychology provides useful insight to guide design.

Section II and III sum up lessons learned from the field of assistive technology for active ageing in general and from previous experiences in robot-based systems, respectively. In section IV useful models from the field of socialpsychology are reviewed on three key processes in assistive robotics: adoption, space sharing and communication. Finally, conclusions are drawn in section V.

\section{Assistive TeChnOlogy For AgING At Home}

\section{A. Technological aids for Quality of Life promotion}

The research field of 'ageing at home' technology focusses on the needs of elders in their daily activities in their own homes. The ageing at home research supports a wider vision of the personal wellbeing systems, and must consider both older adults and their caregivers. In this way a highly specialized support for home-centred health attention extended to all the age groups can be provided [5].

From our point of view, the concept of QoL is a relevant topic in this context, often accessed using two principal axes: autonomy and commitment. Autonomy is referred to as the capability of exercising the control of ones live, selfcare and decision making.; commitment as the state of being and feeling connected, interested in, engaged and compromised with the world and others.

Certain emotions associated to the use of a device can induce undesired effects, no matter how useful the device potentially is. Conversely, certain devices or systems can 
enhance the user's capability perception and therefore enhance his/her real functional capability [3] [4].

Moreover help-giving and receiving is a complex social exchange process that has context dependent rules. Research concerning robotic helpers and assistants emphasizes their potential to improve human performance, but little attention has been given to the effects of robotic helpers on the social and psychological well-being of help recipients. Depending on how and by whom help is given, receiving help might be experienced as threatening (e.g. attempt to self-esteem) or uncomfortable. [6]

\section{B. Acceptance, adoption and effectiveness}

To enhance elderly users' willingness to adopt and use assistive technology (AT) at home, it should be perceived as compatible with their life-style, acceptable by their relatives and caregivers and respectful with the image of how they want their homes to be.

Obtrusiveness- Obtrusiveness is defined as something that is both undesirable and physically or psychologically prominent [7]. As people grow older, they progressively shorten their living space and the immediate environment that is their house becomes more important, feeling more emotional attached to its close environment and more susceptible to changes [4]. In this context the notion of 'users' includes not only 'patients' within their home environments but also their family members and others living in their households.

In tele-health systems, for instance, 8 dimensions in obtrusiveness are identified: the physical dimension, the usability dimension, interference with daily activities, the function dimension, how affects a user's self-concept, the privacy dimension, and worries about whether it could be sustained over time [7].

The role of the stakeholders-The stakeholders have a main influence on the psychosocial process of effective adoption of AT [8] (see Figure 1). This social influence may be on the process of making the decision showing rejection, not recommending it or recommending not using it. Moreover, it may lead to reluctance or willingness for adoption, or it may be indirectly influencing factors involved in the decision making (i.e. intention of use) and behavior (i.e. actual use) of end-users. In the context of AT at home the relevant stakeholders are prescriptors [9], professional careers and informal careers, mainly relatives and friends. In this sense and differently of other treatments or interventions, adoption of new technology-based care and health services implies a change in health providers' practice and in their interaction with patients. Recommendation and adoption of smart technology-based systems is a complex and often difficult collaborative process, [10]mediated by physicians' perceptions of its impact, not only on patient health and wellbeing but also on their own clinical practice.

Opinions and attitudes of health providers could be predicted and managed based on the Technology Adoption Model approach [11], in order to identify the factors involved in remote monitoring systems adoption and adherence [12] [13]. This approach is familiar to technology acceptance modeling, a methodology that will be discussed in section IV.
Opinions about the value and potential of tele-monitoring have been related to two dimensions of the compatibility construct: (i) how well would such a system fit with their patients' needs, capabilities and lifestyle and (ii) how well would such a system fit with their own aspirations and expectations as health professionals. Some skepticism was detected related to the following factors: (i) how accurately and effectively the information drawn from remote sensors would be integrated into clinical reliable and usable information, (ii) patients capability and willingness to adopt an active and efficient role in the process (iii) impact on doctor-patient relationship and (iv) the relative importance of telemedicine implementation compared to other measures to improve patients' assistance [8].

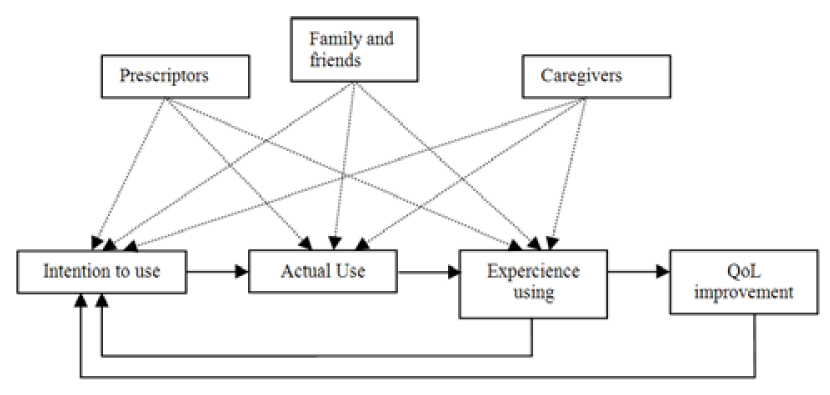

Figure 1. Stakeholders influence on the adoption process

\section{ASSISTIVE SOCIAL ROBOTS FOR SUCCESSFUL AGING}

Several research projects have been carried out with the overall goal of developing mobile robotic solutions assisting elderly people. A major challenge is the integration of robots in smart environments which provide essential context awareness information for the robot by fusing robot sensors with environmental sensors.

\section{A. Smart homes and robot-based assistive services}

In smart home environments, systems are being developed to use sensors in the home to control domestic appliances, such as heating, light dimmers or automatics blinds. Distributed sensors make it possible to cooperatively monitor physical or environmental conditions, such as temperature, sound, vibration, pressure, or motion, and thus build up a more complex representation of the home. A representative example is the 'Peis ecology' [14], combining a large number of sensors with actuators to make assistive systems. Earlier, the smart homes developed at Georgia Tech [15], MIT [16] and Phillips [17] were presented as examples of pervasive sensor systems for domestic environments.

In this context, recognition of human activities is a key topic. This is a broad field of research, ranging from applications in health monitoring to applications in public safety with a plethora of sensors used for activity monitoring. Wearable sensors are used for activity monitoring in applications such as walking, running, and climbing up stairs. Such sensors are usually accelerometers, but full body networks, consisting of a low bandwidth network and tags for communication [18]. 
Lately a lot of research on activity recognition tends to use non-intrusive sensors, based on cameras and computer vision techniques. Comprehensive surveys are given by [19] [20] and more recently [21]. The application areas for these vision systems are mostly surveillance and safety in public domains. The recognition of human actions from cameras is also becoming popular for natural interaction, including gesture recognition for control of games, computers or interaction with robots. Vision cameras have been used in combination with a time of flight range finder [22], which gives valuable extra information for pose estimation. Apart from accurate action recognition, the combined data from range sensor and camera can be used for accurate identification of the person it interacts with.

Also systems that recognize Activities of Daily Living (ADL) from sensor data are now an active topic of research in the field of health monitoring. From simple systems dedicated to recognizing toileting and sleeping behavior to systems able to recognize a large variety of ADLs [23][24][25].

\section{B. Artificial partners for health and wellbeing}

Assistive social robots have been developed and tested to sustain in-clinic and at home rehabilitation and health promotion programs (i.e. lifestyle modification). The used system in these programs acts mainly through social influence providing encouragement, motivation and surveillance for treatment adherence and compliance as well as helping and assisting in daily activities. In this context, the emergence and maintenance of a patient-robot relationship is needed to support long-lasting behavioral change.

\section{Helpers, coaches, companions and pets}

Helpers- A high number of home care robots has been developed in the last few years. To give a few examples: two mobile robotic assistant prototypes, Flo and Pearl, were developed as cognitive reminders and mobile walk-aids to assist elderly with mild cognitive and physical impairments in an assisted living facility [26]. In the context of smart domestic environments, the ROBOCARE project aimed at providing supportive technology for elderly people. Specifically, a domestic environment was equipped with sensors that cooperated with a robot to provide support to the assisted person [27]. In Japan, a robot called uBot-5 is designed as a stable dynamic platform to assist individual in their residential home by providing opportunities for remote monitoring and interaction [28] .The Care-O-Bot, developed by the Fraunhofer Institute, is intended for improving and ensuring QoL for elderly and disabled people. Several modalities for human-robot interaction exist, including vision, speech, lighting, the multipurpose tray with a touch screen for object passing between human and robot.

Coaches- To play the role of a coach, a robot requires knowledge of patient state (physical and psychological) and high level social situated communication skills. The relationship between coach and patient is based on a social bond (affective involvement), a task (i.e. physical exercise) and goals (i.e. functional capability recovery). Obtaining the patient's adherence is an essential issue and requires an agreement about the relevance and usefulness of tasks and goals. Three factors are noted as the most important in creating a relationship with a robot coach: engagement of the user, trust of the system, and motivation to use the system [26][42].

The USC Robotics Lab developed a contact-free socially assistive post-stroke therapeutic robot system [30]. The aim was to assist patients in their daily physical exercise trough social interaction (motivation and feedback) based on its knowledge about the physical exercise of the patient (from sensors in their arms). In Matarić [31] an experiment with post-stroke patients was carried out to investigate how different robot interactive behaviors (from less to more enthusiastic encouragement) and interaction modes (i.e. prerecorded versus synthesized voice) affect patients' willingness to comply with the rehabilitation program and their attitude to the robot. In the SERA [32] study, a subsequent study focused on matching between user's personality and robot's coaching style (from challenging to nurturing) defined by different conditions of social interaction parameters (interaction distances, speed, and vocal content). The reported results provide evidence for users' preference of personality matching.

A robotic weight loss coach was developed at the Media Lab Personal Robots Group [43]. Results of a user study with this robot show that participants using the robot had higher rates of therapy compliance than those using the computer or paper systems. A very new and promising approach in this study was to consider the state of the relationship between user and robot as a key variable to adjust human-robot interaction. The state of the relationship was assessed in a daily bases and labeled as initial, normal or repair.

Pet-like robots- The first efforts towards having socially assistive robotic systems for the elderly have been focused towards constructing robot-pet companions aimed at reducing stress and depression and enhance social interaction and activity. It has been demonstrated that elderly individuals who interact with a seal shaped robot named Paro feel more positive after few interactive sessions with the robot, mainly based on caressing and talking to it [33]. Another robotic companion, the Huggable robot, capable of active relational and effective touch-based interactions with a person, has been developed to increase the wellbeing of hospitalized people [34].

However, in a study concerning robotic coaches with older adults [29] it has been established that not all participants felt comfortable interacting with a robot, some felt too old to play with a toy or did not like animals or do not wish to take on the perceived responsibility for these pet robots..

Companions- In the domain of rehabilitation and healthy life promotion, Tapus [35] carried out a series of trials with senior users ( +70 years old) over 6 months. A humanoid torso robot mounted on a mobile robot base designed for users with dementia and/or Alzheimer's disease, with the main goal of helping users improve or maintain their cognitive attention through encouragement in a music-based cognitive stimulation game. The study focused on the capability of the robot to adapt its behavior to the level of 
disability of the users drawn from the performance of the user and then adjust the level of game challenge. Reported results show that the robot was able to improve or maintain the attention of users and that the robot's capability of adapting its behavior to the individual users' level of disability helped to improve the task performance.

These robots are just a small sample of the platforms often used for research. There is an increasing interest in employing service robots for home use for example HRP2 by Kawada industries, Asimo by Honda, Partner Robots by Toyota, SmartPal by Yaskawa, Emiew by Hitachi, ApriAttenda and ApriAlpha by Toshiba and finally PaPeRobyNEC.

\section{MODELS ON SOCLAL BEHAVIOR TO GUIDE DESIGN}

Some fruitful efforts have been done to adapt models on social behavior to give insight and guide decisions in robotbased systems design for elderly. In this section we outline recent contributions on system adoption, space sharing and proximity, and communication.

\section{A. The Almere acceptance model}

A specific way to approach acceptance of technology is technology acceptance modeling (TAM). It relates the way technology is perceived by a user to the intention to use this technology and assumes that this intention can be taken as an indication of actual use (as is partly illustrated in Figure 2). The perception is modeled by mapping the different perceptive influences.

A technology acceptance model that specifically focusses on assistive social robots for older adults is the Almere model, by Heerink et al. [36] [37]. It incorporates not only factors that are generally used in technology acceptance modeling, like perceived ease of use, trust, perceived usefulness, social influence anxiety and attitude towards technology, but also introduces influences that are specific for assistive social robots for older adults: perceived adaptiveness and social presence.

Perceived adaptiveness has to be interpreted as the extent to which the user thinks a robot will adapt to the users need. In additional research it was established that (1) a proactively adapting robot was favoured above a robot that could be adapted by the user and (2) the user still needed a sense of user control (the robot had to ask for permission before adapting to an established need).

Social Presence is the experience of a social entity when encountering a robot. It was established that a higher sense of social presence could lead to a higher intention to use the robot.

The Almere model goes with a questionnaire that can be used to access either all perceptive aspects or to focus on specific items and test the impact of design features and changes on how the robot is perceived [34].

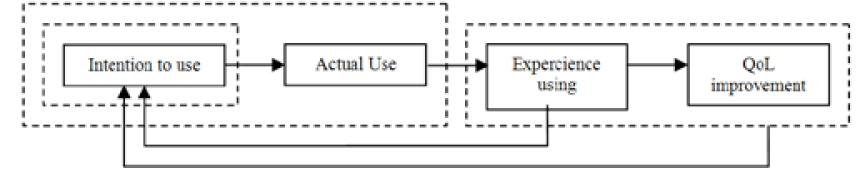

Figure 2. The process of effective assistive technology adoption

\section{B. Smart Space sharing}

A robot at home implies co-presence and sharing the physical space. For robots featured with mobility the questions to be addressed for intelligent space sharing is how to move (i.e. speed, kind of movement and trajectories), where to perform and how to place to do something together.

Assistive robots with "walk-around" functionality get involved in "space relations" with people at home. Space relations are a combination of interpersonal distance and a particular relative position and orientation that occur naturally whenever two or more people engage in and interaction [38]. To be unobtrusive, comfortable and socially congruent smart space relationship has to be carefully designed taking into account social rules of space sharing.

Studies in tele-presence have identified the management of spatial and orientation relationships between people and robot as a main issue in order to improve the quality of interaction. Interpersonal distances convey significant and relevant social information [38]. Optimization of social distance according a specific context is required to keep a desirable level of intimacy that is not simply the physical distance between the agents interacting but a complex joint function of eye-contact, physical proximity, intimacy of topic and smiling [39]. The space formations or spatial patterns (e.g. vis a vis, side-by-side, L-shape, follow or ahead) must be consistent with the roles adopted by the robot, the activities and the spatial constrains, as well as individual variables such as familiarity with the Often the physical constraints of homes (e.g. narrow passages) in combination of navigational or safety requirements could not allow the assistive robot to succeed in maintaining the most acceptable space sharing behavior. In this case it would be interesting to consider compensating this with other robot behavior (e.g. verbally apologising for a inappropriate distance or reduce the eye-contact).

The work of Feil-Seifer and Mataric [40] show how models of interaction based on the social meaning of interpersonal distances can be applied to enhance HRI in a robot-based activity with children with autism. The system was tested in an experimental setting featuring an unobtrusive automatic vision-based assessment of children position. The coded data from several cameras distributed in the environment were feeding the system with relevant online information about children psychological state (e.g. engagement with activity, attitude towards the robot) useful to adjust robot's behavior. This experiment points out the potential of combining "smart home" technologies with socially competent agents.

\section{Communication with social robots}

Co-located social human robot communication is based on a combination of multiple topics, hence multi-modal 
social interaction. Social interaction spans a spectrum of different fields, to give few examples Kidd and Breazeal [29] consider that the ability to look at the user (gaze) for long-term interaction is a key feature. A robot for being social must be capable to express their internal states or emotions either verbally (speech) or non-verbally (e.g. facial expressions, body gestures, sounds, visual cues). There is a large set of basic features necessary to enable social interaction at different levels (e.g. eye contact, face detection and recognition, look-at behaviors, head, arm, and hand gestures, dialogue, tactile displays). On the top of that, a truly personalized robot companion takes into consideration an individual human's likes, dislikes and preferences and adapts its behavior or appearance accordingly [41]. Therefore, a fully autonomous robot capable of effectively communicate in a social manner with a human is an undergoing process.

\section{CONCLUSIONS}

Recently numerous robot-based personal services at home are being developed to assist older people in maintaining their wellbeing. These services usually include monitoring health status, medication management, facilitating communication and engaging users in stimulating activities. In the context of autonomous living the intended outcomes are related to health (e.g. brain fitness games), independence (e.g. helping with housekeeping) and social connectivity.

Some of these services are more efficiently provided by social agents -computer based or robots- able to engage people in social interaction. Robots living at home in a longterm basis offer the added value of more natural and attractive interaction and the possibility to provide new personal services (e.g. company or comfort) based on emotional relational states like trust or affection.

Smart home services facilitated by social robots have showed to be very promising for successful aging though their implementation involves new challenges to address users' functional and emotional needs. To increase the acceptability and sustainability of these systems robot's behaviors must be carefully designed -based on scientific knowledge from social research- to provide them with smart sociability (i.e. respectful to social rules in communication and in space relations).

This social scenario requires updated guidelines for robot's behavior design. A useful tool is the extended models of technology acceptance that -based on empirical data- includes new outcomes (e.g. actual use and clinical impact), new individual variables (e.g. Social Presence, Trust) and a more ecological perspective of the process of effective adoption taking into account stakeholder's perspectives and contextual constrains. Non supportive attitudes such as skepticism towards effectiveness or safety may affect the elderly perceptions on usefulness or effort and may result as well in a lack of involvement to sustain the process.

Moreover, the design of robots' appropriate sociability assisting elderly at home would benefit from lessons learned in other social robotics applications such as tele-presence (i.e. specifications for smart space sharing and social presence) and telemedicine systems (i.e. believes and attitudes of health and care providers towards technologymediated services).

\section{REFERENCES}

[1] F. Alcantud Marín, "Las tecnologías de ayuda: un modelo de intervención," unpublished.

[2] L. J. Oms, J. Cagan, andP. S. Steif, "Exploring the skepticism associated with the use of hip fracture prevention garments," unpublished.

[3] T. Hirsch, J.Forlizzi, E.Hyder, J. Goetz, J. Stroback, and C. Kurtz, "The Elder project: social and emotional factors in the design of eldercare technologies," in 2000 Proc. ACM CUU Conf.

[4] M. Díaz Emociones en el diseño de tecnologías de ayuda en las actividades de la vida diaria para personas mayores, in 2006 Proc. VII Congreso Internacional Interacción Persona-Ordenador, Puertollano, Ciudad Real, 13-17

[5] E. Dishman, "Inventing wellness systems for aging," Computer, vol. 37, no. 5, pp. 34-41, May 2004.

[6] C. Torrey, S.R. Fussell and S. Kiesler, "Trying to be helpful: Social challenges for smart robots" in Workshop-Proceedings of ACM/IEEE Human-Robot Interaction Conference (HRI2008), Amsterdam (pp. 23-26).

[7] K. L. Courtney, G. Demiris, and B. K. Hensel, "Obtrusiveness of information-based assistive technologies as perceived by older adults in residential care facilities: A secondary analysis," Informatics for Health and Social Care, vol. 32, no. 3, pp. 241-249, 2007.

[8] Y. Schikhof, " From acceptance by end-users to added value for all stakeholders," Gerontechnology, vol. 11, no. 2, pp. 238, 2012.

[9] M. Díaz, A.Català, A. Font, L. Narvaiza, and A. Rodríguez-Molinero, "Factors influencing acceptability of ambulatory telemonitoring systems: A qualitative approach of physicians' views as end-users and prescriptors," Gerontechnology, vol. 7, no. 2, 2008.

[10] A. Kintsch, and R. DePaula,"A framework for the adoption of assistive technology," in 2002 Proc. SWAAAC, pp. 1-10.

[11] E. Karahanna, R. Agarwal, and C. Angst, "Reconceptualizing compatibility beliefs in technology acceptance research," MIS Quarterly, vol. 30, 2006.

[12] P. Y. Chau, and P. J. H. Hu, "Information technology acceptance by individual professionals: a model comparison approach," Decision Sciences, vol. 32, no. 4, pp. 699-719, 2001.

[13] C. Y. Chung-Chang, M.Díaz, and C. Angulo, "The impact of introducing therapeutic robots in hospital's organization, "LNCS vol. 7657, pp. 312-315, 2012.

[14] A. Saffiotti, M. Broxvall, M. Gritti, K. LeBlanc, R. Lundh, J. Rashid, B. S. Seo, and Y. J. Cho, "The PEIS-ecology project: vision and results." In 2008 IEEE/RSJ IROS Conf., Nice, France.

[15] C. D. Kidd, R. Orr, G. D. Abowd, C. G. Atkeson, I. A. Essa, B. MacIntyre, E. Mynatt, T. E. Starner, and W. Newstetter, "The aware home: a living laboratory for ubiquitous computing research," Cooperative Buildingsv ol. 1670, pp. 191-198, 1999.

[16] S. S. Intille, K. Larson, E. M. Tapia, J. S. Beaudin, P. Kaushik, J. Nawyn, and R. Rockinson, "Using a live-in laboratory for ubiquitous computing research," in 2006 Proc. Pervasive Computing, pp. 349365 .

[17] B. de Ruyter, and E. Aarts, "Ambient intelligence: visualizing the future," in Proc. 2004 ACM Advanced Visual Interfaces Conf., Gallipoli, Italy.

[18] E. Wade, and H. Asada, "Conductive fabric garment for a cable-free body area network: conductivity analysis for DC power-line communication over fabric media," IEEE Trans. on Pervasive Computing, 2006.

[19] D. M. Gavrila, "The visual analysis of human movement: a survey," Comp. Vision \& Image Unders., vol. 73, no. 1, pp. 82-93, 1999.

[20] T. B. Moeslund, and E. Granum, " A survey of computer vision-based human motion capture," Comp. Vision \& Image Unders., vol.81, no. 3., pp. 231-268, 2001.

[21] P. R. Turaga, V. S. Subrahmanian, and O. Udrea, "Machine recognition of human activities: a survey." IEEE Trans. Circuits and Systems for Video Technology, vol. 18, 2008. 
[22] J. Kubacki, and U. Reiser, "Mirrobot: fast detection of body-parts in the scale-space of range images with an application to human motion copying," in Proc. ROMAN 2006,pp. 552-557.

[23] E. M. Tapia, S. S. Intille, and K. Larson, "Activity recognition in the home using simple and ubiquitous sensors," in Proc. 2004 Pervasive Computing, pp. 158-175.

[24] B. J. A. Krose, T. L. M. v. Kasteren, C. H. S. Gibson, and E. J. v. d. Dool, "Care: context awareness in residences for elderly" Gerontechnology, vol. 7, no. 2, 2008.

[25] M. H. T. Stikic, K. Van Laerhoven, K.; B.Schiele, "ADL recognition based on the combination of RFID and accelerometer sensing," in Proc. 2008 Pervasive Computing Tech. for Healthcare, pp. 258-263

[26] J. Pineau, M. Montemerlo, M. E. Pollack, N. Roy, and S. Thrun, "Towards robotic assistants in nursing homes: challenges and results," Robotics and Autonomous Systems, vol. 42, pp. 273-282, 2003.

[27] A. C. G. Cesta, M. V. Giuliani, F.Pecora, M.Scopelliti, L.Tiberio, "Psychological implications of domestic assistive technology for the elderly," Psychology Journal, vol. 5, no. 3, pp. 229-252, 2007.

[28] P. Deegan, R. Grupen, A. Hanson, E. Horrel, S. Ou, E. Riseman, S. Sen, B. Thibodeau, A. Williams, and D. Xie, "Mobile manipulator for assisted living in residential settings," Autonomous Robots, vol. 24, no. 2, 2008.

[29] C. D. Kidd, and C. Breazeal, "Robots at home: understanding longterm human-robot interaction," in Proc. 2008 IROS Conf.

[30] A.M Okamura, M. J. Mataric, H.I. Christensen, "Medical and healthcare robotics, achievements and opportunities," Robotics and Automation, vol. 17, no.3 pp. 26-37, 2010.

[31] M.J. Matarić, J. Eriksson, D. J. Feil-Seifer, C. J. Winstein, "Socially assistive robotics for post-stroke rehabilitation", Journal of Neuroengineering and Rehabilitation, vol. 4, no. 5, 2007.

[32] Social Engagement with Robots and Agents (SERA), "Literature Review" Available in http://projectsera.eu/publications/deliverables/serad11FINAL.pdf/view 2009

[33] K. S. T. Wada, "Living with seal robots - its socio psychological and physiological influences on the elderly at a care house," Robotics, vol. 23, no. 5, pp. 972-980, 2007.

[34] W. D. Stiehl, J. Lieberman, C. Breazeal, L. Basel, L. Lalla, and M. Wolf," The design of the huggable: A therapeutic robotic companion for relational, affective touch," in AAAI Fall Symposium on Caring Machines :AI in Eldercare, 2009, Washington, USA.

[35] A. Tapus, C. Tapus, and M. J. Mataric, "Assistive Robotics for Customized Cognitive Stimulation and Physical Rehabilitation," inProc. 2009 FSR Conf., Boston, USA.

[36] M. Heerink, B. Kröse, V. Evers, and B. Wielinga, "Measuring acceptance of assistive social agent technology by older adults: the Almere model, "International Journal of Social Robotics, vol. 2, no. 3,pp. 254-268, 2010.

[37] M. Heerink, "How elderly users of a socially interactive robot experience adaptiveness, adaptability and user control," in 12th IEEE 2011 CINTI Conf., pp. 79-84, Budapest, Hungary.

[38] A. Kristoffersson, K. Severinson Eklundh, and A.Loutfi, "Measuring the quality of interaction in mobile robotic telepresence: a pilot's perspective," International Journal of Social Robotics, pp. 1-13, 2012.

[39] M. Argyle, and J. Dean, "Eye-contact, distance and affiliation," Sociometry pp. 289-304, 1965.

[40] D. Feil-Seifer, and M.Mataric, "Distance-based computational models for facilitating robot interaction with children,"Journal of HumanRobot Interaction, 2012.

[41] K. Dautenhahn, "Robots we like to live with?!-A developmental perspective on a personalized, life-long robot companion," in Proc. 3rd IEEE RO-MAN 2004, pp. 17-22, Kurashiki, Japan.

[42] M. Díaz, N. Nuñó, J. Saez-Pons, D. Pardo, C. Angulo. "Building up child-robot relationship for therapeutic purposes: From initial attraction towards long-term social engagement". Automatic Face \& Gesture Recognition and Workshops, IEEE International Conference on, pp. 927-932, 2011.

[43] C. D. Kidd and C. Breazeal. "A Robotic Weight Loss Coach". Twenty-Second Conference on Artificial Intelligence. Vancouver, British Columbia, Canada. AAAI, 2007. 